\title{
Adnexal masses: a severe bilateral compromise case
}

\author{
Piazze Juan $^{1 *}$, Donfrancesco Cristina ${ }^{1,2}$ and Testani Costantino ${ }^{1}$ \\ ${ }^{1}$ Department of Gynecology and Obstetrics, Ospedale SS. Trinità di Sora, Sora (FR), Italy \\ ${ }^{2}$ Departmenf of Experimental Medicine, University of Rome "Sapienza”, Rome (RM), Italy
}

\begin{abstract}
Pelvic inflammatory disease (PID) plays an important role in the evolution of sexually transmitted diseases (STD). The most frequently identified microorganisms are Neisseria gonorrhoeae, Chlamydia trachomatis and Mycoplasma genitalium. The case report presented deals with a severe ominous presentation of bilateral adnexal compromise in a 52 year old woman, and the events that leaded to a bilateral adnexectomy.
\end{abstract}

\section{Introduction}

Pelvic inflammatory disease (PID), is a sexually transmitted infection of the organs of a women's reproductive system, the uterus, ovaries, fallopian tubes, and cervix, caused by a large spectrum of micro-organisms. PID usually develops among sexually active women, especially those with multiple sex partners, although it has also been described among virgins $[1,2]$. The microbiological cause is frequently unknown. In the context of sexually transmitted disease (STD), the most frequently identified microorganisms are Neisseria gonorrhoeae, Chlamydia trachomatis and Mycoplasma genitalium, but bacterial vaginosis and Trichomonas vaginalis can be found too [3]. Symptoms usually include pelvic pain and discomfort during intercourse, accompanied by yellowish vaginal discharge, bleeding between periods and sometimes fever. It is not uncommon that symptoms are mild or absent. The microbiological diagnosis is usually performed on samples of vaginal liquid, endocervix or, when available, surgical specimens. Antibiotic treatment is frequently empirical, started before microbiological culture results; however it should at least cover $\mathrm{N}$. gonorrhoeae, C. trachomatis and M. genitalium, and for most of the authors, anaerobes.

We present one case of severe PID in a patient with pelvic aches and bilateral ovarian masses, our management and some general considerations.

\section{Case report}

A 52-year-old sexually active Caucasian woman, G3 P2012, presented at our attention complaining for pelvic pain. Abdominal examination was unremarkable. There was few yellowish vaginal discharge on speculum and mild cervical motion tenderness on bimanual examination. Her physical findings showed a normal temperature of $37.2^{\circ} \mathrm{C}$, blood pressure $120 / 80 \mathrm{mmHg}$ and pulse 84 beats per minute. Vaginal and cervical cultures for Chlamydia trachomatis, N. gonorrhea and bacterial vaginosis were performed. A pelvic ultrasound scan (Siemens Acuson S2000, HELX Evolution) showed a normal uterus measuring $77 \times 50 \times 48 \mathrm{~mm}$, a moderate amount of fluid in the Douglas pouch and bilateral, multilocular adnexal masses, with moderate flow (color score 3) [4]. Adnexal right mass measured $56 \mathrm{x} 47 \mathrm{~mm}$ and left mass was 55 x $47 \mathrm{~mm}$ (Figures 1 and 2). A complete total body scan (CT scan) revealed a newly formed conglobate pelvic process of $12 \times 9 \mathrm{~cm}$,

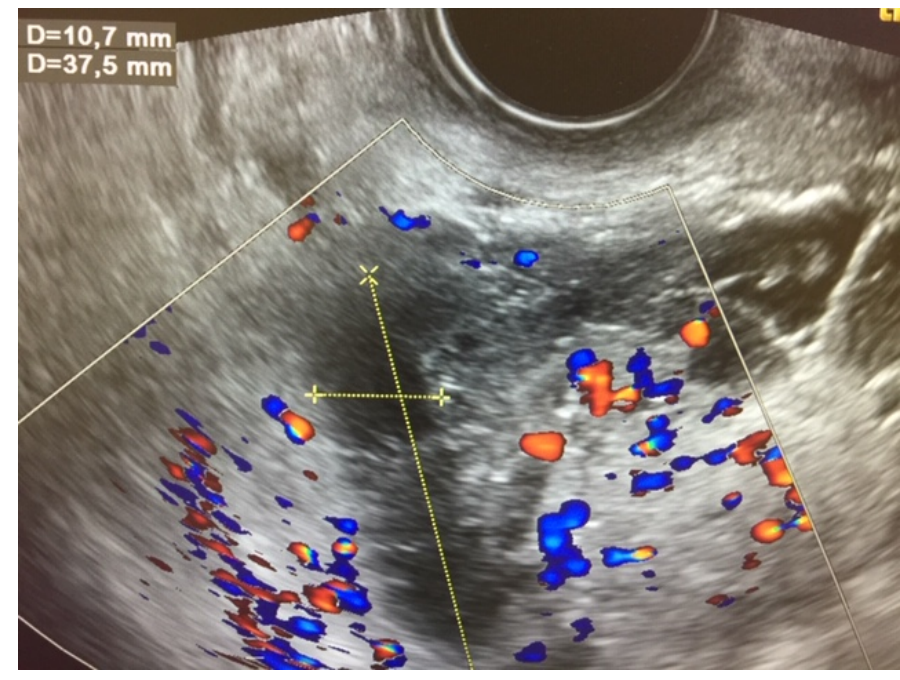

Figure 1. Right adnexal mass with $\mathrm{III}^{\circ}$ Doppler color score present

adherent to uterine wall, in continuity with bladder fund and anterior rectal wall. Moreover, it showed some enlarged pelvic lymph nodes and mesenteric thickening.

We also requested for a full blood count, coagulation studies, C-reactive protein (CRP), human immunodeficiency virus (HIV) serology, HBsAg and started antimicrobial large spectrum therapy with clyndamicin $900 \mathrm{mg}$ intravenous (IV) every 8 hours plus gentamicin IV loading dose $2 \mathrm{mg} / \mathrm{kg}$, followed by a maintenance dose $1.5 \mathrm{mg} / \mathrm{kg}$ every 8 hours. Laboratory tests indicated a mild leukocitosis $\left(12.000 / \mathrm{mm}^{3}\right)$, moderate neutrophilia (73\%), but high CRP (169,3 mg/L [normal range 0.00-5.00]). After three days of antibiotics, CRP started to decrease

${ }^{\star}$ Correspondence to: Piazze Juan, Department of Gynecology and Obstetrics, Ospedale SS. Trinità di Sora, Sora (FR), Italy, E-mail: cloudpia@me.com

Key words: adnexal mass, pelvic inflammatory disease, ultrasound, antibiotics, sexually transmitted disease

Received: October 12, 2018; Accepted: October 19, 2018; Published: October 26,2018 


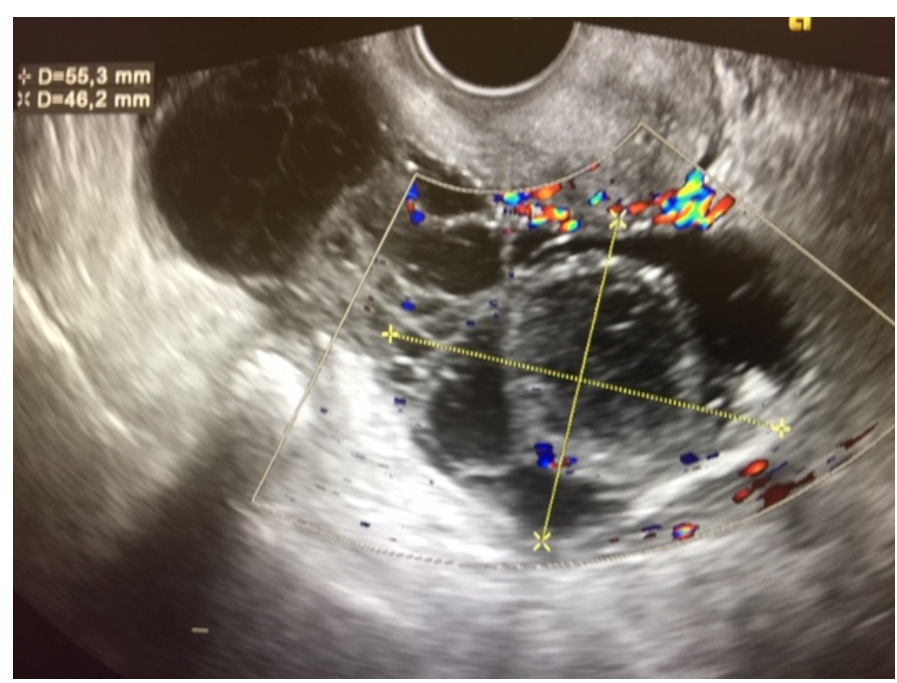

Figure 2. Left adnexal mass with $\mathrm{III}^{\circ}$ Doppler color score present

$(112,6 \mathrm{mg} / \mathrm{L})$, and at 5 th day of therapy it was $51,7 \mathrm{mg} / \mathrm{L}$. The results of vaginal and cervical cultures were positive for Mycoplasma hominis and Ureaplasma parvum.

Laparotomy was performed. Some intestine uterine and tuboovarian adhesions and a little amount of peritoneal fluid were found. Both adnexa were transformed in enlarged cystic masses full of pus and fibrotic phlogistic tissue.

We performed pelvic adhesiolysis, drained abundant purulent secretions and carried out bilateral adnexectomy plus multiple peritoneal and omental biopsies. Histological examination revealed bilateral tuboovarian abscesses, with pus and granulocytic infiltrates. Examination of the peritoneal biopsies and ascites showed inflammation, and Mycoplasma infection was suggested as the aetiology. The patient was discharged from hospital $72 \mathrm{~h}$ after surgery with antibiotic oral therapy and her hospital stay was uneventful.

At follow-up examination four weeks later the patient presented no symptoms.

\section{Comment}

As already mentioned, some women can be asymptomatic or poorly symptomatic. In these situations, when presenting with unilateral or bilateral adnexal masses especially in the age of 40-50 years, differential diagnosis from ovarian malignancies is mandatory.

In this case, the pelvic pain was related to yellowish discharges at admission, nevertheless, a normal temperature was observed. What revealed the character of the patient's status was actually the ultrasound examination, with a severe bilateral voluminous tubarian dilation with third grade vascular appearance at color Doppler examination. The vaginal and cervical cultures showed Mycoplasma hominis and Ureaplasma parvum as a cause of the global infection. Interestingly, the patient had non particular sexual activity, referring a single sexual partner. After five days under large spectrum antimicrobial therapy, the decision to perform an adnexectomy was decided meanly by the mixed microbial infection and the ominous adnexal condition as revealed by ultrasound.

The final comment leads with the long view that a severe PID deserves, with a multifactorial approach (clinical, laboratory analysis, CT scan, antibiotic large spectrum therapy) and the importance of ultrasound technology not only in the diagnosis but in the eventual surgical decision to take.

\section{References}

1. Lareau SM, Beigi RH (2008) Pelvic inflammatory disease and tubo-ovarian abscess Infect Dis Clin North Am 22: 693-708. [Crossref]

2. Kielly M, Jamieson MA (2014) Pelvic inflammatory disease in virginal adolescent females without tubo-ovarian abscess. J Pediatr Adolesc Gynecol 27: e5-e7. [Crossref]

3. Quentin R, Verdon R (2012) Microbiologic basis of diagnosis and treatment of pelvic inflammatory disease. Gynecol Obstet Biol Reprod (Paris) 41:850-863. [Crossref]

4. Timmerman D, Valentin L, Bourne TH, Collins WP, Verrelst H, et al. (2000) Terms, definitions and measurements to describe the sonographic features of adnexal tumors: a consensus opinion from the International Ovarian Tumor Analysis (IOTA) Group. Ultrasound Obstet Gynecol 16:500-505. [Crossref]

Copyright: (C2018 Piazze J. This is an open-access article distributed under the terms of the Creative Commons Attribution License, which permits unrestricted use, distribution, and reproduction in any medium, provided the original author and source are credited. 